\title{
Lie Algebras, 2-Groups and Cotriangular Spaces
}

\author{
Andries E. Brouwer*, Arjeh M. Cohen*, Hans Cuypers*, \\ Jonathan I. Hall ${ }^{\dagger}$, Erik Postma* \\ * Department of Mathematics, Eindhoven University of Technology, \\ P.O. Box 513, 5600 MB Eindhoven, The Netherlands \\ $\dagger$ Department of Mathematics, Michigan State University, \\ East-Lansing, MI, USA
}

August 11, 2008

\begin{abstract}
We describe the construction of a Lie algebra from a partial linear space with oriented lines of size 3, generalizing a construction by Kaplansky. We determine all suitable partial linear spaces and the resulting Lie algebras.
\end{abstract}

\section{Lie oriented partial linear spaces}

A partial linear space is an incidence structure $(P, L)$ with points and lines, such that the point-line incidence graph does not contain quadrangles. Let $(P, L)$ be a partial linear space with lines of size 3 , and let $F$ be a field of characteristic 2 . Construct an algebra $\mathcal{L}:=\mathcal{L}_{F}(P, L)$ with bilinear multiplication on the $F$-vector space $F^{P}$ with basis $P$ by defining the multiplication on the basis:

$$
x y= \begin{cases}z & \text { if } \ell=\{x, y, z\} \in L, \\ 0 & \text { otherwise }(x=y \text { or } x, y \text { noncollinear }) .\end{cases}
$$

In some cases $\mathcal{L}$ is a Lie algebra. This construction was first studied by Kaplansky [8] for symplectic spaces over fields of characteristic 2, and later also by Rotman and Weichsel [10,11]. The general case was studied by Cuypers [4] who found that in characteristic 2 this construction only works in the case described by Kaplansky: a subspace of the partial linear space of hyperbolic lines of a symplectic space.

J. I. Hall suggested that if one associates a cyclic orientation to the lines of the partial linear space, one might obtain Lie algebras over fields of 
arbitrary characteristic. In this paper, we investigate the Lie algebras arising from this construction.

Let $(P, L)$ be a partial linear space with lines of size 3 , where each line $\ell=\{x, y, z\} \in L$ is provided with a cyclic orientation $\sigma(\ell)$, one of the two cyclic permutations $(x, y, z)$ or $(y, x, z)$. Let $F$ be a field. Construct an algebra $\mathcal{L}:=\mathcal{L}_{F}(P, L, \sigma)$ with bilinear multiplication on the $F$-vector space $F^{P}$ with basis $P$ by defining the multiplication on the basis:

$$
[x, y]= \begin{cases}z & \text { if } \ell=\{x, y, z\} \in L \text { and } \sigma(\ell)=(x, y, z) \\ -z & \text { if } \ell=\{x, y, z\} \in L \text { and } \sigma(\ell)=(y, x, z) \\ 0 & \text { otherwise }(x=y \text { or } x, y \text { noncollinear })\end{cases}
$$

We call $\mathcal{L}$ the Kaplansky algebra of the oriented partial linear space $(P, L, \sigma)$ over $F$. This algebra has a bilinear and antisymmetric multiplication, but does not necessarily satisfy the Jacobi identity.

If $\mathcal{L}_{F}(P, L, \sigma)$ is a Lie algebra for some field $F$ of characteristic different from 2 , then $(P, L, \sigma)$ is called a Lie oriented partial linear space and $\sigma$ is called a Lie orientation on $(P, L)$. If $(P, L, \sigma)$ is a Lie oriented partial linear space for some $\sigma$, then we call $(P, L)$ Lie orientable.

\section{$1.1 \quad$ The examples}

A partial linear space is called connected when its point-line incidence graph is connected. As we shall see below, the connected Lie orientable partial linear spaces are of four types, that we describe here.

(a) $\mathcal{T}\left(\Omega, \Omega^{\prime}\right)$, the partial linear space obtained from two disjoint sets $\Omega, \Omega^{\prime}$ by taking as points the subsets $A$ of $\Omega \cup \Omega^{\prime}$ with $|A \cap \Omega|=2$, and as lines the triples $\{A, B, C\}$ of points, where $A+B+C=0$ in the binary vector space $2^{\Omega \cup \Omega^{\prime}}$.

(b) $\mathcal{S} p(V, B)$, the partial linear space obtained from a binary vector space $V$ provided with a symplectic form $B$ by taking as points the vectors outside the radical of $B$, and as lines the hyperbolic lines.

(c) $\mathcal{O}(V, Q)$, the partial linear space obtained from a binary vector space $V$ provided with a quadratic form $Q$ by taking as points the vectors where $Q$ is nonzero and that lie outside the radical of $B$, the symplectic form associated to $Q$, and as lines the elliptic lines.

(d) $P V \backslash P W$, the partial linear space obtained by taking, for a binary vector space $V$ with subspace $W$ of codimension 3, the points of $P V$ (the projective space associated to $V$ ) that are not in $P W$, and the lines of $P V$ that are disjoint from $P W$. Here $P W$ can be empty. 


\section{$1.2 \quad$ Flipping}

Let $p \in P$. Define $\sigma_{p}$ by

$$
\sigma_{p}(\ell)= \begin{cases}\sigma(\ell) & \text { if } p \notin \ell, \\ (\sigma(\ell))^{-1} & \text { if } p \in \ell .\end{cases}
$$

Then $\mathcal{L}_{F}(P, L, \sigma)$ and $\mathcal{L}_{F}\left(P, L, \sigma_{p}\right)$ are isomorphic by the algebra isomorphism that maps $p$ to $-p$ and fixes all other basis elements. So, if we simultaneously reverse the orientation of all lines in $L$ through one point $p$, we end up with an isomorphic algebra. This operation will be called flipping at $p$. Similarly, we can define flipping at a subset $S$ of $P$ as the operation that reverses the orientation of a line $l$ exactly $|l \cap S|$ times. Note that these automorphisms of the algebra generate an elementary Abelian 2-group.

Two orientations of $(P, L)$ are called flipping equivalent, if one can be obtained from the other by applying a flipping.

\subsection{Main result}

Our main result is the following theorem.

Theorem 1.1 Let $(P, L, \sigma)$ be a connected Lie oriented partial linear space. Then $(P, L)$ is isomorphic to one of the spaces (a) $\mathcal{T}\left(\Omega, \Omega^{\prime}\right)$, (b) $\mathcal{S} p(V, B)$, (c) $\mathcal{O}(V, Q)$, (d) $P V \backslash P W$ as described above. Conversely, each of these spaces is Lie orientable, and admits up to flipping a unique Lie orientation $\sigma$. The resulting Kaplansky algebra $\mathcal{L}_{F}(P, L, \sigma)$ is a Lie algebra, provided that in case (d) the field $F$ has characteristic 3.

The families (a)-(c) also occur in Kaplansky's original construction. Part of the results of this note were given earlier in [9].

\section{Binary orthogonal geometries}

A subspace of a partial linear space $(P, L)$ is a subset $S$ of the point set with the property that each line meeting $S$ in at least 2 points is contained in $S$. We often identify a subspace $S$ with the partial linear space with point set $S$ whose lines are the lines of $(P, L)$ contained in $S$.

If $(P, L)$ is equipped with an orientation then $S$ is naturally equipped with an orientation. One easily checks that the Kaplansky algebra of a subspace of $(P, L)$ is isomorphic to the subalgebra of the Kaplansky algebra generated by the points of the subspace.

In this section we prove that the partial linear spaces of types (a), (b), and (c) are Lie orientable. As each of the spaces in (a) and (b) is isomorphic to a subspace of some partial linear space $\mathcal{O}(V, Q)$ of type (c), see for example [7], it suffies to handle case (c). 
Let $\Pi=(P, L)$ be the partial linear space $\mathcal{O}(V, Q)$ and let $\phi: V \rightarrow \mathbb{F}_{2}$ be a bilinear form with the property that $\phi(v, v)=Q(v)$ for all $v \in V$. For $\ell=\{x, y, z\} \in L$ define $\sigma=\sigma_{\phi}$ by

$$
\sigma(\ell)= \begin{cases}(x, y, z) & \text { if } \phi(x, y)+\phi(y, z)+\phi(z, x)=0 \\ (x, z, y) & \text { if } \phi(x, y)+\phi(y, z)+\phi(z, x)=1\end{cases}
$$

As a line $l \in L$ consists of a triple of nonsingular vectors, we have $\phi(x, y)+$ $\phi(y, x)=\phi(x+y, x+y)+\phi(x, x)+\phi(y, y)=1$ so that $\sigma$ is well-defined.

Since $x+y+z=0$ we have $\phi(x, x)+\phi(x, y)+\phi(x, z)=0$ so that $\phi(x, y)=\phi(y, z)=\phi(z, x)$.

The multiplication on the Kaplansky algebra $\mathcal{L}_{F}(P, L, \sigma)$ can now be expressed as follows. For any $u, v \in P$ we have

$$
[u, v]=Q(u+v) \cdot(-1)^{\phi(u, v)} \cdot(u+v) .
$$

Lemma 2.1 The Kaplansky algebra $\mathcal{L}_{F}(P, L, \sigma)$ is a Lie algebra for every field $F$. In particular, $\sigma$ is a Lie orientation on $\Pi$.

Proof. Let $F$ be a field. To prove the lemma, it suffices to check that the Jacobi identity holds in the Kaplansky algebra $\mathcal{L}_{F}(P, L, \sigma)$.

Let $u, v, w$ be three points in $P$. Then

$$
\begin{aligned}
{[[u, v], w] } & =\left[Q(u+v) \cdot(-1)^{\phi(u, v)} \cdot(u+v), w\right] \\
& =Q(u+v+w) \cdot(-1)^{\phi(u+v, w)} \cdot Q(u+v) \cdot(-1)^{\phi(u, v)}(u+v+w) \\
& =Q(u+v+w) \cdot Q(u+v) \cdot(-1)^{\phi(u, v)+\phi(v, w)+\phi(w, u)+1}(u+v+w) .
\end{aligned}
$$

Inside the subspace of $V$ generated by $u, v$ and $w$ we easily check that $Q(u+v+w)=0$ or $Q(u+v)+Q(v+w)+Q(w+u)=0$, and the Jacobi identity $[[u, v], w]+[[v, w], u]+[[w, u], v]=0$ follows.

\section{$2.1 \quad E_{2}$-groups}

The above construction of $\sigma$ is closely related to the construction of extraspecial groups and the following generalization of orthogonal space, see [6].

Definition 2.2 A group $E$ is called an $E_{2}$-group if it is a 2-group and it has a normal subgroup $Z$ of order 2 such that $E / Z$ is elementary Abelian. $Z$ is called the scalar subgroup of $E$.

Indeed, given a binary quadratic space $(V, Q)$ and bilinear form $\phi: V \rightarrow$ $\mathbb{F}_{2}$ with $\phi(v, v)=Q(v)$ we can equip the set $E=V \times \mathbb{F}_{2}$ with the following multiplication for $(v, \epsilon)$ and $(w, \eta)$ in $E$ :

$$
(v, \epsilon) \cdot(w, \eta)=(v+w, \epsilon+\eta+\phi(v, w)) .
$$


This multiplication turns $E$ into an $E_{2}$-group with scalar subgroup $Z=$ $\{0\} \times \mathbb{F}_{2}$. Hall [6] shows that, up to isomorphism, every $E_{2}$-group can be obtained in this way.

The cyclic subgroups of order 4 and quaternion subgoups of order 8 of $E$ are in one-one correspondence (and hence can be identified) with the points and lines, respectively, of $\mathcal{O}(V, Q)$. This partial linear space is therefore called the geometry of $E$. The orientation on this geometry can be recovered by fixing a generator of the form $(v, 0)$ for each point in $E$. Indeed, if $p, q$ and $r$ are three collinear points, with generators $\tilde{p}=(v, 0), \tilde{q}=(u, 0)$ and $\tilde{r}=(w, 0)$, then the orientation of the line equals $(p, q, r)$ if $\tilde{p} \tilde{q}=\tilde{r}$ and $(p, r, q)$ otherwise.

Given an $E_{2}$-group $E$, we can identify the Kaplansky Lie algebra with a subalgebra of the Lie algebra defined on the group algebra of $E$. To this end we use the following construction by Plesken as described in [2].

Definition 2.3 Let $G$ be a finite group. For $g \in G$, denote the element $g-g^{-1}$ of the group algebra $F[G]$ by $\hat{g}$. We call $\mathcal{L}_{F}(G):=\langle\hat{g} \mid g \in G\rangle$, with Lie bracket $[\hat{g}, \hat{h}]:=\hat{g} \hat{h}-\hat{h} \hat{g}$, the Plesken Lie algebra of $G$ over $F$.

The multiplication expands to

$$
[\hat{g}, \hat{h}]=\widehat{g h}-\widehat{g h^{-1}}-\widehat{g^{-1} h}+\widehat{g^{-1} h^{-1}},
$$

so $\mathcal{L}_{F}(G)$ is linearly spanned by the elements $\hat{g}$. Clearly $\hat{g}=0$ if and only if $g=g^{-1}$. Furthermore, $\hat{g}=-\widehat{g^{-1}}$. If $S$ is a set of algebra elements $\hat{g}$ where $S$ does not contain $\widehat{g^{-1}}$ for any $\hat{g} \in S$, then its elements are linearly independent: $F[G]$ can be decomposed into subspaces $\left\langle g, g^{-1}\right\rangle_{F}$ that are all linearly independent, and the elements of $S$ are nonzero and in different such subspaces.

We turn our attention to the Plesken Lie algebra of an $E_{2}$-group $E$. The nonzero generators are those $\hat{g}$ where $g$ has order 4 . If $e$ is an element of order 4 , then $\hat{e}=(1-z) e$. Note that $1-z$ is a central element of the group algebra, and

$$
(1-z)^{2}=1-2 z+z^{2}=2(1-z) .
$$

Since $z e=e^{-1}$, we also have $z \hat{e}=e^{-1}-e=-\hat{e}$. Furthermore, we can obtain a basis of $\mathcal{L}_{F}(E)$ by taking the algebra elements corresponding to a set of representatives for the points (i.e., subgroups of order 4 ) of $E$. Let $R$ be such a set of representatives. If $e, f$ and $g$ are three elements of $R$ with $e f=z^{\alpha} g$, then

$[\hat{e}, \hat{f}]=(1-z)^{2}(e f-f e)=(1-z)^{2}\left(z^{\alpha} g-z^{1+\alpha} g\right)=z^{\alpha}(1-z)^{3} g=4 \cdot(-1)^{\alpha} \hat{g}$.

Since the dimensions of $\mathcal{L}_{F}(E)$ and the Kaplansky algebra of the oriented partial linear space of $E$ are both equal to half the number of elements of $E$ of order 4 , we have proven the following proposition: 


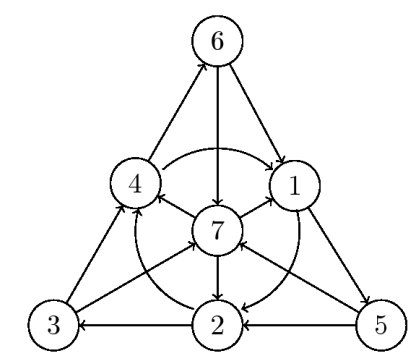

Figure 3.1: Oriented Fano plane

Proposition 2.4 Let $E$ be an $E_{2}$-group. Choose a set $R$ of representatives for the points of $E$. The map from the Plesken Lie algebra of $E$ to the Kaplansky algebra of the geometry of $E$ with orientation obtained from $R$, given by $\tilde{e} \mapsto 4 \hat{e}$ for $e$ in $R$, where $\hat{e}$ is the element of the Kaplansky algebra corresponding to $e$, is an isomorphism if $\operatorname{char} F \neq 2$.

If $\operatorname{char} F=2$ and we take $E$ as in the proposition, then the regular multiplication on the subalgebra $\langle e Z \mid e \in R\rangle_{F}$ of $F[E / Z]$ turns out to satisfy the Jacobi identity. This subalgebra is then isomorphic to the Kaplansky algebra of the oriented partial linear space of $E$. This is easily checked by hand.

\section{Octonions and projective geometries}

In this section we describe a class of Lie algebras arising as Kaplansky algebras from the geometry of points and lines of a binary projective space missing some fixed codimension 3 space.

We start with the Fano plane. It is well known that the 7 lines of the Fano plane can be oriented in such a way that the corresponding Kaplansky algebra over a field $F$ is isomorphic to the algebra of split octonions over $F$ modulo its center $F \cdot 1$, e.g. see [1].

Indeed, if the 7 points of the Fano plane are called $e_{1}, \ldots, e_{7}$ then the octonions can be described as the 8-dimensional algebra over $F$ with basis $1, e_{1}, \ldots, e_{7}$ and multiplication defined by the orientation as given in Figure 3 .

This orientation encodes the following multiplication table. 


\begin{tabular}{l|llllllll}
$\times$ & 1 & $e_{1}$ & $e_{2}$ & $e_{3}$ & $e_{4}$ & $e_{5}$ & $e_{6}$ & $e_{7}$ \\
\hline$e_{1}$ & $e_{1}$ & -1 & $e_{4}$ & $e_{7}$ & $-e_{2}$ & $e_{6}$ & $-e_{5}$ & $-e_{3}$ \\
$e_{2}$ & $e_{2}$ & $-e_{4}$ & -1 & $e_{5}$ & $e_{1}$ & $-e_{3}$ & $e_{7}$ & $-e_{6}$ \\
$e_{3}$ & $e_{3}$ & $-e_{7}$ & $-e_{5}$ & -1 & $e_{6}$ & $e_{2}$ & $-e_{4}$ & $e_{1}$ \\
$e_{4}$ & $e_{4}$ & $e_{2}$ & $-e_{1}$ & $-e_{6}$ & -1 & $e_{3}$ & $e_{7}$ & $-e_{5}$ \\
$e_{5}$ & $e_{5}$ & $-e_{6}$ & $e_{3}$ & $-e_{2}$ & $-e_{7}$ & -1 & $e_{1}$ & $e_{4}$ \\
$e_{6}$ & $e_{6}$ & $e_{5}$ & $-e_{7}$ & $e_{4}$ & $-e_{3}$ & $-e_{1}$ & -1 & $e_{2}$ \\
$e_{7}$ & $e_{7}$ & $e_{3}$ & $e_{6}$ & $-e_{1}$ & $e_{5}$ & $-e_{4}$ & $-e_{2}$ & -1
\end{tabular}

It is a straightforward task to check that over fields $F$ of characteristic 3 the quotient of the octonions by its center satisfies the Jacobi identity. In particular, the Fano plane is Lie orientable.

Next, let $V$ be a binary vector space and let $W$ be a subspace of $V$ of codimension 3. Let $P V$ and $P W$ be the corresponding projective spaces. Let $P V \backslash P W$ denote the geometry of points and lines of $P V$ disjoint from $P W$. Fix a subspace $F_{0}$ of the partial linear space $P V \backslash P W$ isomorphic to the Fano plane. Provide $F_{0}$ with a Lie orientation $\sigma_{0}$. Then $\sigma_{0}$ can be extended to a Lie orientation $\sigma$ on $P V \backslash P W$ in the following way.

Each point $p$ of $P V \backslash P W$ is equal or noncollinear to a unique point in $F_{0}$ which we denote by $\bar{p}$. If $l=\{p, q, r\}$ is a line then $\bar{l}=\{\bar{p}, \bar{q}, \bar{r}\}$ is a line in $F_{0}$. For each line $l=\{p, q, r\}$ of $P V \backslash P W$ define $\sigma(l)$ to be the cycle $(p, q, r)$ if and only if $\sigma(\bar{l})=(\bar{p}, \bar{q}, \bar{r})$.

It is now straightforward to check that $\sigma$ is a Lie orientation. So, we have proved the following.

Proposition 3.1 Suppose $V$ is a binary vector space and $W$ a subspace of codimension 3 in $V$. Then $\Pi=P V \backslash P W$ admits a Lie orientation such that for fields of characteristic 3 the corresponding Kaplanksy algebra is a Lie algebra.

\section{Partial linear spaces with a Lie orientation}

In the previous sections we showed that the partial linear spaces of types (a)-(d) are Lie orientable. In this section we show that no other connected partial linear spaces are Lie orientable.

Let $\Pi=(P, L)$ be a connected partial linear space admitting a Lie orientation $\sigma$, such that for the field $F$ of characteristic $\neq 2$ the Kaplansky algebra $\mathcal{L}_{F}(P, L, \sigma)$ is a Lie algebra.

As the intersection of subspaces of $(P, L)$ is again a subspace, we can define the subspace generated by a subset $X$ of $P$ to be the intersection of all subspaces containing $X$. A subspace generated by (the union of) two intersecting lines is called a plane. 
Proposition 4.1 All planes of $\Pi$ are isomorphic to a dual affine plane or a Fano plane.

Proof. Let $\{a, b, c\}$ and $\{a, d, e\}$ be two lines. We may assume that their orientations are $(a b c)$ and $(a d e)$. By the Jacobi identity

$$
[[b, c], d]+[[c, d], b]+[[d, b], c]=e+[[c, d], b]+[[d, b], c]=0 .
$$

Any product of standard basis elements is either 0 or plus or minus a standard basis element, and therefore, so are the second and third terms of this sum. Hence, either one of the terms is $-e$ and the other is 0 , or both are $e$ and $\operatorname{char} F=3$.

Case 1: Suppose $[[c, d], b]=-e$ and $[[d, b], c]=0$ (the case where these values are switched is symmetric). Then $c$ and $d$ must be collinear; call the third point on that line $f=[c, d]$ (the case where $f=-[c, d]$ leads to an isomorphic oriented partial linear space). Then $b, f$ and $e$ must be on a line as well, with $[b, f]=e$. The plane containing only the points and lines mentioned so far is the dual affine plane of order 2 , and with this choice for $\sigma$ it satisfies the Jacobi identity.

If, in addition to the aforementioned points and lines, we have $b \sim d$, then the third point on that line (say $g=[b, d]$ ) cannot be collinear to $c$. The Jacobi identity on $g, d$ and $a$ then requires that $\{a, g, f\}$ is a line, oriented (agf). This violates the Jacobi identity on $a, b$ and $d$. Hence the dual affine plane of order 2 is the only plane that can lead to a Lie algebra for fields with characteristic other than 3.

Case 2: Now suppose $\operatorname{char} F=3$ and $[[c, d], b]=[[d, b], c]=e$. We cannot have that $[c, d]= \pm[d, b]$; for, if that would be the case, then by the axiom of partial linear spaces, $c=b$. So let $f=[c, d]$ and $g=[d, b]$ (again, the cases where $f=-[c, d]$ and / or $g=-[d, b]$ lead to isomorphic oriented partial linear spaces). The Jacobi identity on $a, b$ and $d$ then requires that $[a, g]=f$. This is the last line of the Fano plane. No more lines can be added, since each point is already collinear with every other point. This plane, depicted in Figure 3, also satisfies the Jacobi identity. The orientation on the lines is the same as is often used to define the octonions, see e.g. [1].

Proposition 4.2 Let $(P, L, \sigma)$ be an oriented partial linear space. Then $\sigma$ is a Lie orientation if and only if for every plane of $(P, L)$, the restriction of $\sigma$ to the plane induces a Lie orientation on this plane.

Proof. Let $\left(P^{\prime}, L^{\prime}\right)$ be a plane of $(P, L)$. Clearly $\mathcal{L}_{F}\left(P^{\prime}, L^{\prime},\left.\sigma\right|_{L^{\prime}}\right)$ is a subalgebra of $\mathcal{L}$. Hence, it is certainly necessary for $\mathcal{L}$ to be a Lie algebra that $\mathcal{L}_{F}\left(P^{\prime}, L^{\prime},\left.\sigma\right|_{L^{\prime}}\right)$ be a Lie algebra. To show sufficiency, suppose that every $\mathcal{L}_{F}\left(P^{\prime}, L^{\prime},\left.\sigma\right|_{L^{\prime}}\right)$ is a Lie algebra for planes $\left(P^{\prime}, L^{\prime}\right)$. Let $p, q, r \in P$. We will 
show that the Jacobi identity holds for $p, q$ and $r$. If there are no two intersecting lines containing $p, q$ and $r$, then the Jacobi identity certainly holds. Otherwise, they are contained in a plane; then the Jacobi identity holds because of the assumption. So the Jacobi identity holds on all triples of basis elements. Since the Jacobi identity is linear, we are done.

Proposition 4.1 gives us a powerful tool to analyze the partial linear spaces that can be Lie oriented. Indeed, the connected partial linear spaces in which any two lines generate a dual affine of Fano plane have been classified. They come in two families. The spaces containing only dual affine planes are called cotriangular. They have been classified by J. I. Hall [7] and are the spaces as described in cases (a), (b) and (c) of Theorem 1.1. The partial linear spaces containing at least one Fano plane are the spaces $P V \backslash P W$ obtained by removing the points and lines of a projective space $P V$, for $V$ a binary vector space, that meet a proper subspace $W$ of $V$ nontrivially. This follows from the results of Hale [5] and Cuypers [3].

The following lemma shows that if the space $P V \backslash P W$ is Lie orientable, then the subspace $W$ has codimension at most 3 .

Lemma 4.3 Let $\operatorname{dim} V=4$. Then $P V$ has no Lie orientation.

Proof. Suppose the partial linear space $(P, L)$ of points and lines in the 4-dimensional vector space over $\mathbb{F}_{2}$ has a Lie orientation $\sigma$. Let $\ell=\{a, b, c\}$ and $m=\{x, y, z\}$ be two disjoint lines in $P V$ with $\sigma(\ell)=(a, b, c)$ and $\sigma(m)=(x, y, z)$. Let $a x$ denote the third point on the line on $a$ and $x$, and similarly for the other pairs of points in $\ell \times m$. We have now named all 15 points. By potentially flipping w.r.t. $a x$, we may assume that $\sigma(\{x, a x, a\})=$ $(x, a x, a)$, and similarly for the 8 other lines $k$ connecting $m$ to $\ell$ : we assume the orientation is such that $\sigma(k)$ maps $k \cap \ell$ to $k \cap m$.

We define projections $\pi_{\ell}: P \backslash m \rightarrow \ell$ and $\pi_{m}: P \backslash \ell \rightarrow m$, mapping the point $p q$, with $p \in \ell$ and $q \in m$, to $p$ or to $q$ respectively (and fixing $\ell$ and $m$, respectively). If a plane $\Pi$ contains $m$ or $\ell$, then we will see that the restriction of $\sigma$ to $\Pi$ is fully determined by the choices that we have made, as follows. Take for $\Pi$ the plane containing $\ell$ and $x$. The Jacobi identity on $a, c$ and $x$ tells us that

$$
-b x+[c x, a]+[a x, c]=0 ;
$$

since all three of these terms are $\pm b x$, we find $[c x, a]=[a x, c]=-b x$. This determines the value of $\sigma$ on the lines $\{c x, b x, a\}$ and $\{c, b x, a x\}$. The Jacobi identity on $a, b$ and $x$ additionally gives the value on $\{c x, b, a x\}$. We see that $\pi_{\ell}$ is orientation-reversing on these lines.

The same is true if we take for $\Pi$ a different plane containing $\ell$, and if we take for $\Pi$ a plane containing $m$, then $\pi_{m}$ is orientation-reversing on the lines in there as well. 
Now take a plane containing only one point of both $\ell$ and $m$, e.g. the plane containing $a, x$ and $b y$. The Jacobi identity on these three points cannot be satisfied anymore:

$[[a, x], b y]+[[x, b y], a]+[[b y, a], x]=[a x, b y]-[b z, a]+[c y, x]=[a x, b y]= \pm c z$.

Using the results of Hall [7], M.P. Hale, Jr. [5] in the finite case and Theorem 1.1 of [3] we obtain the classification part of Theorem 1.1: any connected Lie oriented partial linear space is of one of the four types givem there.

\section{Uniqueness of the Lie orientation}

In this section we prove that each of the partial linear spaces in the conclusion of Theorem 1.1 admits, up to flipping, a unique Lie orientation.

Let $\Pi$ be one of the partial linear spaces of Theorem 1.1. An even collection of lines of $\Pi$ is a set of lines that covers each point an even number of times.

Proposition 5.1 In $\Pi$ any finite even collection of lines is the sum (in the binary vector space $2^{L}$ ) of even collections of size four.

Proof. As any finite set of even lines is contained in a finite subspace of $\Pi$, we can assume $\Pi$ to be finite and use induction on the the number of points of $\Pi$. Let $H$ be a (geometric) hyperplane of $\Pi=(P, L)$ (arbitrary, or to be chosen later). Given a finite even collection $\mathcal{C}$ of lines in $\Pi$, we show that $\mathcal{C}$ is the sum of an even collection in $H$ and some even collections of size 4.

The lines in $L$ not contained in $H$ become edges of a graph $G$ on the complement of $H$, and the collection $\mathcal{C}$ becomes the set of edges of a subgraph $C$ of $G$ that has even degree at each vertex. If $C$ can be triangulated (i.e., is a sum of triangles in $G$ ), then we are done, since a triangle $x, y, z$ in $G$ is contained in a plane of $\Pi$ meeting $H$ in a line, and hence derived from lines $\{a, x, y\},\{b, y, z\}$, and $\{c, z, x\}$ with $a, b, c \in H$, where $\{a, b, c\}$ is a line. So, the set of three lines of such a triangle is the sum of an even 4 -set and a line contained in $H$.

So, it remains to prove that the graph $C$ can be triangulated.

Induction on the size of $C$. We distinguish cases (a)-(d) as in the classification of Theorem 1.1.

In case (d), $P G(V \backslash W)$, choose $H$ a hyperplane not containing $W$. The points outside $P G(W)$ fall into at least 7 cosets, and two points are joined by an edge when they lie in distinct cosets. Given a cycle $\ldots \sim w \sim x \sim y \sim$ $z \sim \ldots$ we can pick $p$ outside $H$ in a coset not containing $w, x, y, z$ and see 
that the cycle is the sum of the shorter cycle $\ldots \sim w \sim p \sim z \sim \ldots$ and the triangles $w, x, p$ and $x, y, p$ and $y, z, p$. Repeating this proces will eventually provide a triangulation of the cycle.

In case (a), $T\left(\Omega, \Omega^{\prime}\right)$, first suppose $|\Omega|>3$. Let $\alpha \in \Omega$, and let $H$ be the hyperplane of points not containing $\alpha$. The complement of $H$ consists of the points $A$ with $\alpha \in A$, and two points $A, A^{\prime}$ with $A \cap \Omega=\{\alpha, \beta\}$ and $A^{\prime} \cap \Omega=\left\{\alpha, \beta^{\prime}\right\}$ are adjacent when $\beta \neq \beta^{\prime}$. Given a cycle without diagonals $\ldots \sim w \sim x \sim y \sim z \sim \ldots$ we have $\beta, \gamma \in \Omega$ with $w \cap \Omega=y \cap \Omega=\{\alpha, \beta\}$ and $x \cap \Omega=z \cap \Omega=\{\alpha, \gamma\}$. Now the point $p=\{\alpha, \delta\}$ for some $\delta$ distinct from $\alpha, \beta, \gamma$ lies outside $H$ and is adjacent to each of $w, x, y, z$. As in the previous case, this proves that $C$ can be triangulated.

Next suppose $|\Omega|=3$. Now the collinearity graph of the partial linear space is complete tripartite. Let $H$ be the hyperplane of points not containing $\alpha^{\prime} \in \Omega^{\prime}$. Given a cycle without diagonals $\ldots \sim w \sim x \sim y \sim z \sim \ldots$ the points $w, x, y, z$ must lie in two of the three parts of the tripartition, and we can pick a point $p$ adjacent to $w, x, y, z$ in the third part.

In case (b), $\mathcal{S} p(V, B)$, two points $x, y$ are joined by an edge when $B(x, y)=$ 1. Put $R:=\operatorname{Rad}(B)$. Then $\operatorname{dim} V / R$ is even. If $\operatorname{dim} V / R=2$ or 4 , we are in case $\mathrm{T}\left(\Omega, \Omega^{\prime}\right)$ with $|\Omega|=3$ or 6 , which was treated already. So assume $\operatorname{dim} V / R \geq 6$. Pick a point $a$ and put $H=a^{\perp}$. Since $a$ is in an even number of lines of $\mathcal{C}$, we can add even 4 -sets to $\mathcal{C}$ in order to remove all lines that pass through $a$. Given a cycle $\ldots \sim w \sim x \sim y \sim z \sim \ldots$ in $C$, we can find a point $p$ in the complement of $H$ adjacent to each of $w, x, y, z$ when $a$ does not lie in the span of $w, x, y, z$. Suppose $a$ lies in the span of $w, x, y, z$. If $a=w+x+y+z$ then $B(x, a)=0$, which yields a contradiction. Hence $a=w+z$ and $B(w, z)=1$. Since we had removed all lines on $a$ from $\mathcal{C}$, the edge $w z$ is a diagonal in the cycle. This shows that if $x_{0} \sim x_{1} \sim \ldots \sim x_{g-1} \sim x_{0}$ is a shortest cycle in $C$, then we can make $C$ smaller by adding triangles, unless $g=6$ and $a=x_{0}+x_{3}=x_{1}+x_{4}=x_{2}+x_{5}$. But now modulo even sets of size 4 the sum of the lines on the edges of this 6 -cycle is zero.

In case $(\mathrm{c}), \mathcal{O}(V, Q)$, we have the subspace of the previous example induced by the points $x$ with $Q(x)=1$. In low dimensions this space is one of the examples seen already. (Let $Q$ be the set of projective points $x$ with $Q(x)=0$, and put $S=R \cap Q$ so that $Q$ induces a nondegenerate quadratic form on $V / S$. We may assume that $Q$ is nonempty. If $\operatorname{dim} V / S=2$, then $Q$ is hyperbolic and we have $P G(n, 2)-P G(-1,2)$. If $\operatorname{dim} V / S=3$, then we have $T\left(4, \Omega^{\prime}\right)$. If $\operatorname{dim} V / S=4$, then either $Q$ is hyperbolic, and we have a disconnected space (two copies of $T\left(3, \Omega^{\prime}\right)$ ), or $Q$ is elliptic, and we have $T\left(5, \Omega^{\prime}\right)$. If $\operatorname{dim} V / S=5$, then we have $T\left(6, \Omega^{\prime}\right)$. If $\operatorname{dim} V / S=6$ and $Q$ is hyperbolic, then we have $T\left(8, \Omega^{\prime}\right)$.)

So assume $\operatorname{dim} V / S \geq 6$. Again remove all lines through some point $a$ 
(with $Q(a)=1$ ), and take $H=a^{\perp}$. Consider a cycle $\ldots \sim w \sim x \sim y \sim$ $z \sim \ldots$ in $C$. If it has length 6 and opposite vertices sum to $a$, then as before modulo even sets of size 4 the sum of the lines on the edges of this 6-cycle is zero. Otherwise, for suitable choice of $w$, we have $a \neq w+z$, and then $a$ is independent from $w, x, y, z$. If $w, x, y, z$ are dependent, then they lie in a plane, and the three edges $w x, x y, y z$ can be replaced by the single edge $w z$. So we may assume that $a, w, x, y, z$ are independent. Desired: a point $p$ with $Q(p)=1$ and $B(p, a)=B(p, w)=B(p, x)=B(p, y)=B(p, z)=1$. If $\operatorname{dim} V / S \geq 7$ then we can restrict attention to a nondegenerate subspace of dimension 7 and satisfy the conditions on $B(p,$.$) there, and then satisfy$ $Q(p)=1$ by adding the nucleus to $p$, if necessary. So we may assume that $\operatorname{dim} V / S=6$ and $Q$ is elliptic. Now there is a unique point $p$ that satisfies the conditions, as one sees by explicit inspection.

Corollary 5.2 Up to flipping, the Lie orientation of the partial linear space $\Pi$ is unique.

Proof. From the classification in Theorem 1.1 we see that each Lie orientable partial linear space is the union of an increasing sequence of finite subspaces, and hence we may assume that the partial linear space $\Pi$ is finite.

We may also assume that $\Pi$ is connected. If $\Pi$ consists of a single plane then the statement is true, as one verifies directly.

Let $N$ be the point-line incidence matrix of $\Pi$. Consider two Lie orientations of $\Pi$. Let $x$ be the binary row vector indexed by $L$ with $x_{\ell}=1$ when the two orientations differ on $\ell$. We have to show that $x$ is in the row span of $N$, given that the restriction of $x$ to the set of lines in any plane is in the row span of $N$ restricted to that same set of lines.

To this end, it suffices to show that if $y$ is a binary row vector orthogonal to the row space of $N$, then $y$ is the sum of such vectors that are zero outside the set of lines in a plane. In other words, if we have an even collection of lines, then that collection is the sum of even collections contained in a plane.

In the dual affine plane there is only one nonempty such collection, the set of all four lines. In the Fano plane any such collection is the set of four lines missing a some point.

The above corollary finishes the proof of Theorem 1.1.

\section{The structure of the Kaplansky algebras}

In this section, we will determine the structure and isomorphism types of the Kaplansky Lie algebras that we have constructed earlier.

Let $\Pi=(P, L, \sigma)$ be a Lie oriented partial linear space. For $p, q \in P$ we write $p \equiv q$ if and only if $p^{\perp}=q^{\perp}$. The partial linear space $\Pi$ is called reduced if and only if all 三-equivalence classes are trivial, i.e. consist of a single point. 
Lemma 6.1 Suppose that $\Pi$ is connected and reduced. Let $F$ be a field such that the Kaplansky algebra $\mathcal{L}_{F}(\Pi)$ is a Lie algebra. Then $\mathcal{L}_{F}(\Pi)$ is simple.

Proof. Let $I$ be a nontrivial ideal of $\mathcal{L}_{F}(\Pi)$. If $I$ contains a point $p \in P$, then by connectedness of $\Pi$ it contains all points of $P$ and thus is equal to $\mathcal{L}_{F}(\Pi)$. Thus assume that $P \cap I=\emptyset$ and pick $p_{1}, \ldots, p_{n} \in P$ with $n>1$ minimal such that $0 \neq x=\alpha_{1} p_{1}+\cdots+\alpha_{n} p_{n} \in I$ for some $\alpha_{1}, \ldots, \alpha_{n} \in F$. Since $p_{1} \not \equiv p_{n}$, we can assume, up to permuting 1 and $n$, that there is a $p \in P$ collinear with $p_{1}$ but not with $p_{n}$. But then $[p, x]=\alpha_{1}\left[p, p_{1}\right]+\alpha_{2}\left[p, p_{2}\right]+\cdots+\alpha_{n}\left[p, p_{n}\right]=$ $\alpha_{2}\left[p, p_{2}\right]+\cdots+\alpha_{n}\left[p, p_{n}\right] \in I$. Since $\left[p, p_{i}\right]$ is either $0,+q$ or $-q$ for some point $q \in P$, we find a contradiction with the minimality of $n$. Hence, if $I \neq 0$, then it equals $\mathcal{L}_{F}(\Pi)$, proving simplicity of $\mathcal{L}_{F}(\Pi)$.

Now suppose $\Pi$ is connected but reduced. By the classification result Theorem 1.1 it follows that we can find a subspace $\Pi_{0}=\left(P_{0}, L_{0}, \sigma_{0}\right)$ of $\Pi$ meeting each $\equiv$-class in just one point.

The orientation $\sigma_{0}$, the restriction of $\sigma$ to $L_{0}$, can be extended to an orientation $\hat{\sigma}$ on the whole of $\Pi$ in the following way. If $l=\{x, y, z\}$ is a line in $L$, then there exists unique line $l^{\prime}=\left\{x^{\prime}, y^{\prime}, z^{\prime}\right\}$ in $\Pi_{0}$ such that $x^{\prime} \equiv x$, $y^{\prime} \equiv y$ and $z^{\prime} \equiv z$. Now let $\sigma^{\prime}(l)=(x, y, z)$ if and only if $\sigma\left(l^{\prime}\right)=\left(x^{\prime}, y^{\prime}, z^{\prime}\right)$. By 4.2 it is straightforward to check that this orientation $\hat{\sigma}$ is a Lie orientation. As all Lie orientations are flipping equivalent, and we can, to analyze the structure of the corresponding Kaplansky algebra, assume that $\hat{\sigma}=\sigma$. But then the two subspaces

$$
I_{-}=\left\langle p-p^{\prime} \mid p \equiv p^{\prime} \in P\right\rangle
$$

and

$$
I_{+}=\left\langle p+p^{\prime} \mid p \equiv p^{\prime} \in P\right\rangle
$$

are clearly ideals of $\mathcal{L}_{F}(\Pi)$.

In particular we have proved

Proposition 6.2 Suppose $\Pi$ is a Lie oriented partial linear space, $F$ a field and $\mathcal{L}_{F}(\Pi)$ the Kaplansky Lie algebra of $\Pi$ over $F$.

Then $\mathcal{L}_{F}(\Pi)$ is simple if and only if $\Pi$ is reduced.

We investigate the structure of $\mathcal{L}_{F}(\Pi)$ somewhat further.

Clearly, if $F$ is of characteristic 2, then $I_{-}=I_{+}$is Abelian. From now on we assume that the characteristic of $F$ is not 2 . Let $\Pi_{1}$ be a subspace of $\Pi$ meeting each $\equiv$-class in all but one point. Such subspaces exist. Indeed, we can obtain $\Pi_{1}=\left(P_{1}, L_{1}\right)$ by intersecting $P$ with an appropriate hyperplane of the projective space in which $\Pi$ naturally embeds. As above, we can easily check that

$$
J_{-}=\left\langle p-p^{\prime} \mid p \equiv p^{\prime}, p \in P_{1}, p^{\prime} \notin P_{1}\right\rangle
$$


and

$$
J_{+}=\left\langle p+p^{\prime} \mid p \equiv p^{\prime}, p \in P_{1}, p^{\prime} \notin P_{1}\right\rangle
$$

are ideals of $\mathcal{L}_{F}(\Pi)$. Moreover, if we extend $F$ with $\sqrt{2}$, then over this extension $J_{-}, J_{+}$and $\mathcal{L}_{F}\left(\Pi_{1}\right)$ are isomorphic. Indeed, the map

$$
\begin{gathered}
P_{1} \rightarrow J_{ \pm} ; \\
p \mapsto \frac{1}{2} \sqrt{2}\left(p \pm p^{\prime}\right),
\end{gathered}
$$

induces an isomorphism from $\mathcal{L}_{F}\left(\Pi_{1}\right)$ to $J_{ \pm}$. Repeating this process we obtain the following.

Proposition 6.3 Suppose $\Pi$ is a Lie oriented partial linear space, $F$ a field and $\mathcal{L}_{F}(\Pi)$ the Kaplansky Lie algebra of $\Pi$ over $F$.

If the characteristic of $F$ is not 2, then, possibly after extending $F$ by $\sqrt{2}$, the Kaplansky Lie algebra $\mathcal{L}_{F}(\Pi)$ is a direct sum of pairwise commuting simple Kaplansky Lie algebras all isomorphic to $\mathcal{L}_{F}\left(\Pi_{0}\right)$.

For the remainder of this section we will consider the case where $\Pi$ is reduced and the Kaplansky Lie algebra is simple and identify the Lie algebras with some classical Lie algebras. We start with the following result:

Theorem 6.4 Let $F$ be a field of characteristic different from 2 and $\Pi=$ $(P, L, \sigma)$ a finite reduced Lie oriented partial linear space with associated Kaplansky Lie algebra $\mathcal{L}=\mathcal{L}_{F}(\Pi)$. Then $\mathcal{L}$ is a classical Lie algebra isomorphic to

(a) $\mathfrak{s l}\left(2^{n}, F\right)$ if $(P, L)$ is isomorphic to $\mathcal{S} p(V, f)$ for some nondegenerate binary symplectic space $(V, f)$ of dimension $2 n$.

(b) $\mathfrak{s o}\left(2^{n}, F\right)$ if $(P, L)$ is isomorphic to $\mathcal{O}(V, f)$ for some nondegenerate binary orthogonal space $(V, f)$ of dimension $2 n$ and maximal Witt index.

(c) $\mathfrak{s p}\left(2^{n-1}, F\right)$ if $(P, L)$ is isomorphic to $\mathcal{O}(V, f)$ for some nondegenerate binary orthogonal space $(V, f)$ of dimension $2 n$ and Witt index $n-1$.

Proof. First consider the case that $(P, L)$ is isomorphic to $\mathcal{O}(V, Q)$, where $(V, Q)$ is a nondegenerate binary quadratic space of dimension $2 n$ and maximal Witt index. The $E_{2}$-group $E=E(V, Q)$ is then isomorphic to the subgroup $E(n) \leq \mathrm{GL}_{2^{\mathrm{n}}}(F)$ defined as

$$
E(n)=E(1)^{\otimes n}=E(1) \otimes \cdots \otimes E(1),
$$

where

$$
E(1)=\left\langle\left(\begin{array}{ll}
0 & 1 \\
1 & 0
\end{array}\right),\left(\begin{array}{cc}
1 & 0 \\
0 & -1
\end{array}\right)\right\rangle
$$


Denote by $\mathcal{L}_{F}(E)$ the Plesken Lie algebra over $F$ of $E$. Let $\psi: E \rightarrow E(n)$ be an isomorphism and $\bar{\psi}: \mathcal{L}_{F}(E) \rightarrow \mathfrak{g l}\left(2^{n}, F\right)$ the linear map defined by $\bar{\psi}(\hat{e})=\psi(e)-\psi\left(e^{-1}\right)$ for all $e \in E$. Then $\bar{\psi}$ is a Lie algebra morphism and the Plesken algebra and hence also the Kaplansky Lie algebra defined by $E$ is isomorphic to the image of $\bar{\psi}$. Notice that the elements of order 4 in $E(n)$ are represented by skew-symmetric matrices. So, by construction $\bar{\psi}$ maps all elements of $E$ into elements of $\mathfrak{s o}\left(2^{n}, F\right)$. Moreover, as $\mathcal{L}_{F}(E)$ and $\mathfrak{s o}\left(2^{n}, F\right)$ have the same dimension, we find these Lie algebras to be isomorphic. This proves statement (b).

Now consider the element

$$
J_{n}:=\left(\begin{array}{cc}
0_{n-1} & I_{n-1} \\
-I_{n-1} & 0_{n-1}
\end{array}\right)
$$

of $E(n)$ where $0_{m}$ and $I_{m}$ represent the $2^{m} \times 2^{m}$ zero and identity matrix, respectively, and let $e$ be its preimage in $E$, Then centralizer of $e$ in $\mathcal{L}_{F}(E)$ contains the Plesken algebra corresponding to a subspace $\Delta$ of $\Pi$ isomorphic to $\mathcal{S} p(W, f)$ for some binary $2(n-1)$-dimensional nondegenerate symplectic space $(W, f)$.

The centralizer of $J_{n}$ in $\mathfrak{s o}\left(2^{n}, F\right)$ consists of all matrices of the form $\left(\begin{array}{cc}A & B \\ -B & A\end{array}\right)$ with $A=-A^{\top}$ and $B=B^{\top}$. The latter centralizer is isomorphic to $\mathfrak{g l}\left(2^{n-1}, F\right)$ and hence modulo its center to $\mathfrak{s l}\left(2^{n-1}, F\right)$. (Indeed, mapping $\left(\begin{array}{cc}A & B \\ -B & A\end{array}\right)$ to $A+B$ yields an isomorphism.) As $\mathcal{L}_{F}(\Delta)$ and $\mathfrak{s l}\left(2^{n-1}, F\right)$ have the same dimension, they are isomorphic, proving (a).

Next consider the element

$$
\left(\begin{array}{ll}
0_{n-1} & J_{n-1} \\
J_{n-1} & 0_{n-1}
\end{array}\right)
$$

in $\mathfrak{s o}\left(2^{n}, F\right)$ and its preimage $f$ in $E$. The centralizer of $e$ and $f$ in the Plesken algebra contains the Plesken algebra corresponding to a subspace of $\Pi$ isomorphic to $\mathcal{O}\left(W, Q^{\prime}\right)$ for some nondegenerate binary quadratic space $\left(W, Q^{\prime}\right)$ of dimension $2 n-2$ and of Witt index $n-2$. This space is mapped by $\bar{\psi}$ into the subalgebra of $\mathfrak{s o}\left(2^{n}, F\right)$ consisting of all matrices of the form $\left(\begin{array}{cc}A & B \\ -B & A\end{array}\right)$ with $A=-A^{\top}$ and $B=B^{\top}$, and $(A+B) J_{n-1}=J_{n-1}(A+B)^{\top}$. As the latter is isomorphic with $\mathfrak{s p}\left(2^{n-1}, F\right)$ and the dimensions fit, we have proved (c).

It remains to identify the Lie algebras arising form the Fano plane and from cotriangular of the form $\mathcal{T}(\{1, \ldots, n\}, \emptyset))$. We first identify some Cartan subalgebras. 
Lemma 6.5 Any subspace of $\mathcal{L}_{F}(P, L, \sigma)$ spanned by an inclusion wise maximal set of basis elements corresponding to noncollinear points is a Cartan subalgebra.

Proof. Let $H$ be such a subspace. Since the multiplication restricted to $H$ is zero, $H$ is certainly an Abelian subalgebra. Denote the set of points corresponding to the generators of $H$ by $U$, and write $\tilde{p}$ for the Lie algebra element corresponding to the point $p$.

Let $u \in U$ and suppose $\left[\sum \alpha_{i} \tilde{v}_{i}, \tilde{u}\right] \in H$. Suppose $v_{i} \notin U$ and $v_{i} \sim u$. Denote the third point on the line connecting $v_{i}$ and $u$ by $w$. Note that $w \notin$ $U$, since $u$ is already an element of $U$. Then $\left[\tilde{v}_{i}, \tilde{u}\right]= \pm \tilde{w} \notin H$. Furthermore, different basis vectors $\tilde{v}_{j}$ cannot "compensate" this element outside $H$, since for $i \neq j$, the product $\left[\tilde{v}_{j}, \tilde{u}\right]$ is a multiple of a different standard basis vector. So $\alpha_{i}=0$. Since every $v_{i} \notin U$ is collinear to some point in $U$ (otherwise $U$ would not be maximal), $\sum \alpha_{i} \tilde{v}_{i} \in H$. Hence $H$ is self-normalizing. Thus it is a Cartan subalgebra.

Theorem 6.6 $\mathcal{L}_{F}(\mathcal{T}(\{1, \ldots, n\}, \emptyset))$ is of Chevalley type $B_{k}$ if $n=2 k+1$ and of type $D_{k}$ if $n=2 k$.

Proof. Take the subalgebra $H$ spanned by the elements $h_{\ell}:=\{\widetilde{2 \ell-1,2 \ell}\}$ where $\ell \leq k$, and where, like in the proof of Lemma 6.5, the tilde turns a point into the corresponding algebra element. This spanning set satisfies the requirements of Lemma 6.5, so $H$ is a Cartan subalgebra. For $\ell<m \leq k$ and for $\alpha, \beta \in\{ \pm 1\}$, let us define

$r_{\ell, m}^{\alpha, \beta}=\{2 \ell-\widetilde{1,2 m}-1\}+\alpha \sqrt{-1}\{2 \ell \widetilde{-1,2} m\}+\beta \sqrt{-1}\{2 \ell \widetilde{, 2 m}-1\}-\alpha \beta\{\widetilde{2 \ell, 2 m}\}$.

Furthermore, if $n$ is odd, then for $\ell \leq k$ and $\alpha \in\{ \pm 1\}$, we additionally define

$$
r_{\ell}^{\alpha}=\{2 \widetilde{\ell-1, n}\}+\alpha \sqrt{-1} \widetilde{\{2 \ell, n\}} .
$$

Now

$$
\left[h_{\ell}, r_{\ell, m}^{\alpha, \beta}\right]=-\beta \sqrt{-1} r_{\ell, m}^{\alpha, \beta}, \quad\left[h_{m}, r_{\ell, m}^{\alpha, \beta}\right]=-\alpha \sqrt{-1} r_{\ell, m}^{\alpha, \beta}, \quad\left[h_{\ell}, r_{\ell}^{\alpha}\right]=-\alpha \sqrt{-1} r_{\ell}^{\alpha},
$$

and all other products between elements of the form $h_{*}$ on the one hand, and elements of the form $r_{*}$ on the other hand, are zero. Thus we define $\left\{e_{\ell}\right\}$ to be the ordered basis of $H^{*}$ dual to the basis of $H$ consisting of the elements $\sqrt{-1} h_{\ell}$; then the projective point containing $r_{\ell, m}^{\alpha, \beta}$ acts as $\beta e_{\ell}+\alpha e_{m}$ and $r_{\ell}^{\alpha}$, if present, acts as $\alpha e_{\ell}$. These root systems are of type $D_{k}$ and $B_{k}$ if $n$ is even or odd, respectively.

Theorem 6.7 Suppose $\Pi=(P, L, \sigma)$ is the Fano plane equipped wit a Lie orientation $\sigma$ and $F$ a field of characteristic 3. Then, after possibly adding $\sqrt{-1}$ to $F$, the Kaplansky algebra $\mathcal{L}_{F}(\Pi)$ is isomorphic to $A_{2}(F)^{\prime}$, the commutator subalgebra of the Lie algebra of type $A_{2}$ over $F$. 
Proof. Take any point $p \in P$ as a generator for a Cartan subalgebra; if $\{p, q, r\} \in L$ and $p^{\sigma(\ell)}=q$, then $q+\sqrt{-1} r$ and $q-\sqrt{-1} r$ span eigenspaces of $p$. These eigenspaces form the images of a set of root spaces under modding out the centre. This finishes the proof.

\section{References}

[1] John C. Baez, The octonions, Bull. Amer. Math. Soc. (N.S.) 39 (2002), no. 2, 145-205 (electronic).

[2] Arjeh M. Cohen and Donald Taylor, On a certain Lie algebra defined by a finite group, preprint February 2005, to appear in Amer. Math. Monthly.

[3] Hans Cuypers, On delta spaces satisfying Pasch's axiom, J. Geom. 53 (1995), no. 1-2, 67-75.

[4] Hans Cuypers, Lie algebras and cotriangular spaces, Bull. Belg. Math. Soc. Simon Stevin 12 (2005), no. 2, 209-221.

[5] Mark P. Hale, Jr., Finite geometries which contain dual affine planes, J. Combinatorial Theory Ser. A 22 (1977), no. 1, 83-91.

[6] Jonathan I. Hall, The number of trace-valued forms and extraspecial groups, J. London Math. Soc. (2) 37 (1988), no. 1, 1-13.

[7] Jonathan I. Hall, Graphs, geometry, 3-transpositions, and symplectic $\mathbf{F}_{2}$-transvection groups, Proc. London Math. Soc. (3) 58 (1989), no. 1, 89-111.

[8] Irving Kaplansky, Some simple Lie algebras of characteristic 2, Lie algebras and related topics (New Brunswick, N.J., 1981), Lecture Notes in Math., vol. 933, Springer, Berlin, 1982, pp. 127-129.

[9] Erik Postma, From Lie Algebras to Geometry and Back, Ph. D. Thesis, TUE, Eindhoven, 2007.

[10] Joseph J. Rotman, Projective planes, graphs, and simple algebras, J. Algebra 155 (1993), no. 2, 267-289.

[11] J. J. Rotman and P. M. Weichsel, Simple Lie algebras and graphs, J. Algebra 169 (1994), no. 3, 775-790. 